\title{
Study on the Degradation of Dye Pollutants Over Silver Phosphate Photocatalyst
}

\author{
Tiantian $\mathrm{Wu}^{1, \mathrm{a}}$, Yanping Liang ${ }^{1, \mathrm{~b}}$ and Fangyuan Deng ${ }^{1, \mathrm{c}}$ \\ ${ }^{1}$ School of Advanced materials and Nano-technology , Xidian University, Xi'an 710126, China \\ a1207441288@qq.com, btianniu6107@163.com, cmaydayfy@163.com
}

Keywords: $\mathrm{Ag}_{3} \mathrm{PO}_{4}$, Photocatalytic degradation, Methylene blue

Abstract. The study deals with the visible-light-driven photodegradation of dye pollutants, especially for methylene blue(MO) over silver phosphate photocatalyst. The $\mathrm{Ag}_{3} \mathrm{PO}_{4}$ photocatalyst was synthesized by a simple ultrasonic-assisted ion-exchange method in weak acidity solution including silver nitrate, sodium dihydrogen phosphate, and sodium phosphate . X-ray diffractometer (XRD), scanning electron microscope (SEM) were carried out to characterize the as-obtained samples. As-synthesized photocatalyst exhibited an excellent photocatalytic efficiency. Under visible light irradiation, nearly $100 \%$ of methylene blue $\left(10 \mathrm{mg} \cdot \mathrm{L}^{-1}, 50 \mathrm{~mL}\right)$ can be degraded by only $0.040 \mathrm{~g}$ photocatalyst in 25 minutes. The influence of dye initial concentration and recyclability was also explored.

\section{Introduction}

In recent years, photocatalytic technology which applied in water treatment to the degradation of dye pollutants attracts more and more widely attention[1]. $\mathrm{TiO}_{2}$-based photocatalyst has been widely used in the photodegradation of dyes, which has the advantages of high activity, good stability, and low price[2]. However, $\mathrm{TiO}_{2}$ photocatalyst with the large bandgap $(3.2 \mathrm{eV})$ can only utilize the UV light, which greatly limits the utilization of solar energy[3-5]. For utilizing solar energy more fully, it is indispensable to design new visible-light-driven photocatalysts[6].

In this area, $\mathrm{Ag}_{3} \mathrm{PO}_{4}$ photocatalyst was found to be an active visible-light-driven photocatalyst and has a huge potential to use solar energy[7,8]. $\mathrm{Ag}_{3} \mathrm{PO}_{4}$ with a bandgap of $2.4 \mathrm{eV}$ has an exceptionally high photocatalytic efficiency due to the high separation of photoexcited electrons and holes[8-10]. In this work, the $\mathrm{Ag}_{3} \mathrm{PO}_{4}$ photocatalyst was successfully prepared in weak acidity solution. The photocatalytic efficiency of the as-synthesized product was investigated by photodegradation of different dyes, especially for methylene blue. In addition, the influence of dye initial concentration and recyclability was also explored.

\section{Experimental section}

$\mathrm{Ag}_{3} \mathrm{PO}_{4}, \mathrm{Na}_{3} \mathrm{PO}_{4}$, and $\mathrm{NaH}_{2} \mathrm{PO}_{4}$ were purchased from Xi'an Chemical Reagent Factory $(\mathrm{China})$. TiO was obtained from Tianjin Hongyan Chemical Reagent Factory(China). Methylene blue and methyl orange(MO) were obtained from Nankai Chemical Plant (China). All of the reagents in our experiments were used as received.

A simple ion-exchange method was employed to prepare $\mathrm{Ag}_{3} \mathrm{PO}_{4}$ product at room temperature. $0.003 \mathrm{~mol}$ of $\mathrm{AgNO}_{3}$ was dissolved in $60 \mathrm{~mL}$ water, and $0.001 \mathrm{~mol}$ of the different proportion of $\mathrm{NaH}_{2} \mathrm{PO}_{4}$ and $\mathrm{Na}_{3} \mathrm{PO}_{4}$ were dissolved in $30 \mathrm{~mL}$ water. Then, the phosphate solution was added by drops into the above solution under ultrasonic oscillations. After another $15 \mathrm{~min}$, the yellow precipitate was collected by filtration, washed with distilled water and dried in vacuum drying oven at $60^{\circ} \mathrm{C}$ for six hours.

The phases of the as-prepared products were characterized by X-ray diffraction (XRD) under a $\mathrm{D} / \mathrm{X}-2700 \mathrm{X}$-ray diffractometer (China) employing $\mathrm{Cu} \mathrm{K} \alpha$ radiation, $\lambda=0.15406 \mathrm{~nm}$. The scanning electron microscope (SEM, JSM-6360) was carried out to characterize the morphology and size of the products . 
The photocatalytic efficiency of the as-obtained particles were evaluated through the degradation of dyes (MB and MO) under visible light irradiation. An LED lamp with a light intensity of 0.042 $\mathrm{W} \cdot \mathrm{cm}^{-2}$ was used as visible light source. $0.040 \mathrm{~g}$ photocatalyst was added into an aqueous dye solution $\left(10 \mathrm{mg} \cdot \mathrm{L}^{-1}, 50 \mathrm{~mL}\right)$. Before light irradiation, the suspension was stirred in the dark for 15 min. Approximately $5 \mathrm{~mL}$ of the suspension was taken at given time intervals and separated through centrifugation (3000 rpm, $5 \mathrm{~min}$ ). The concentration of dyes was analyzed on a visible spectrophotometer (722S, China).

\section{Results and discussion}

Fig.1 shows the XRD patterns of as-synthesized photocatalysts $\mathrm{Ag}_{3} \mathrm{PO}_{4}$. As shown in Fig.1, all of the diffraction peaks can be well-indexed to the cubic structure of $\mathrm{Ag}_{3} \mathrm{PO}_{4}(\mathrm{JCPDS}$ No.: 06-0505), and it was not observed characteristic peak for other types, indicating that the high purity of $\mathrm{Ag}_{3} \mathrm{PO}_{4}$ product was formed.

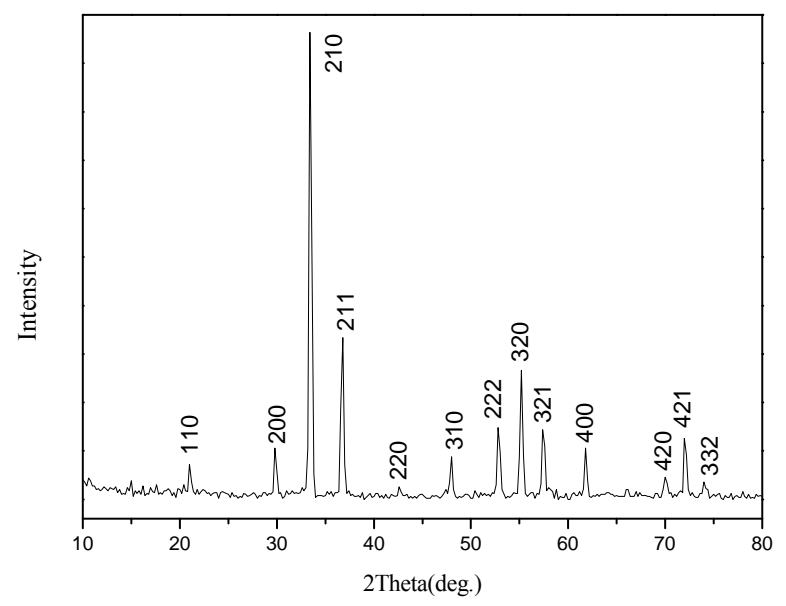

Fig.1. XRD pattern of the as-obtained particles

The morphologies of the as-prepared particles were analyzed by SEM. As shown in Fig.2, $\mathrm{Ag}_{3} \mathrm{PO}_{4}$ particles obtained in weak acidity solution(Fig.2c) were homogeneous in distribution and small in size compare with that obtained in neutral solution(Fig.2b). Fig.2a indicate that the $\mathrm{Ag}_{3} \mathrm{PO}_{4}$ prepared in alkaline solution has partly decomposed to $\mathrm{Ag}$.
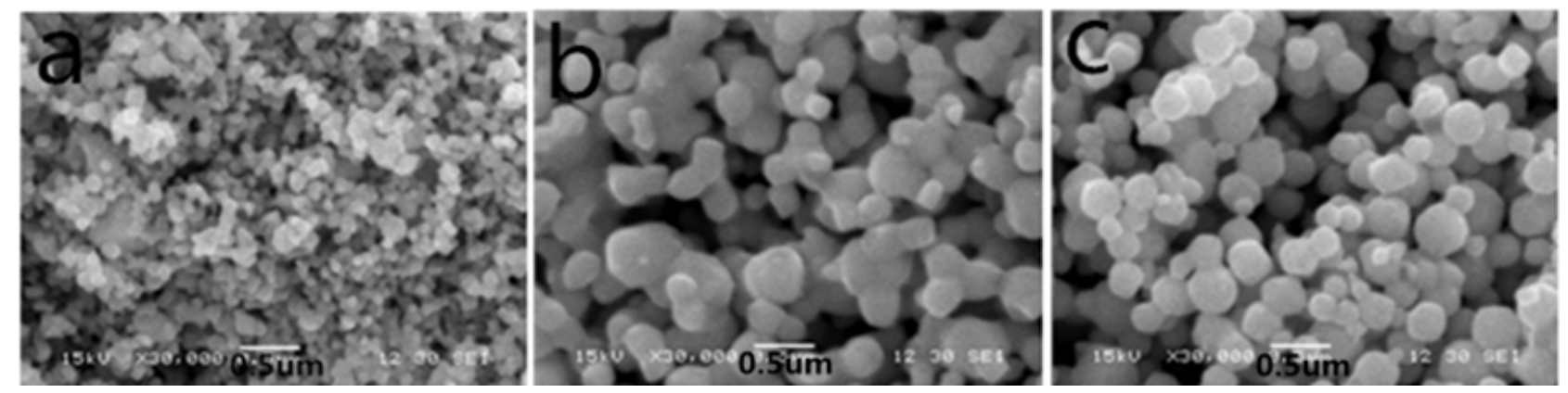

Fig.2. SEM micrographs of the as-synthesized particles

The photocatalytic activities of the as-prepared were evaluated by the degradation of the typical dyes (MB, MO) in water. As shown in Fig.3, $\mathrm{Ag}_{3} \mathrm{PO}_{4}$ products exhibited superior photocatalytic activity in the photodegradation of $\mathrm{MB}$ under visible light, as compared to the $\mathrm{TiO}_{2}$. It is obvious that, the $\mathrm{Ag}_{3} \mathrm{PO}_{4}$ prepared in weak acidity solution has the best photocatalytic activity due to its homogeneous distribution and small size.

Fig. 4 shows the influence of the initial MB concentration on the degradation rate over $\mathrm{Ag}_{3} \mathrm{PO}_{4}$ catalyst. When the concentration of dyes is very low, the degradation process can be well matched 
with pseudo-first-order kinetics obtained from Langmuir-Hinshelwood model[11]. The pseudo-first -order kinetics equation is as Eq. 1,

$$
\ln \left(C_{0} / C_{t}\right)=k t \text {. }
$$

It can be seen that the photodegradation rate of $\mathrm{MB}$ is much higher when the initial concentration is lower.

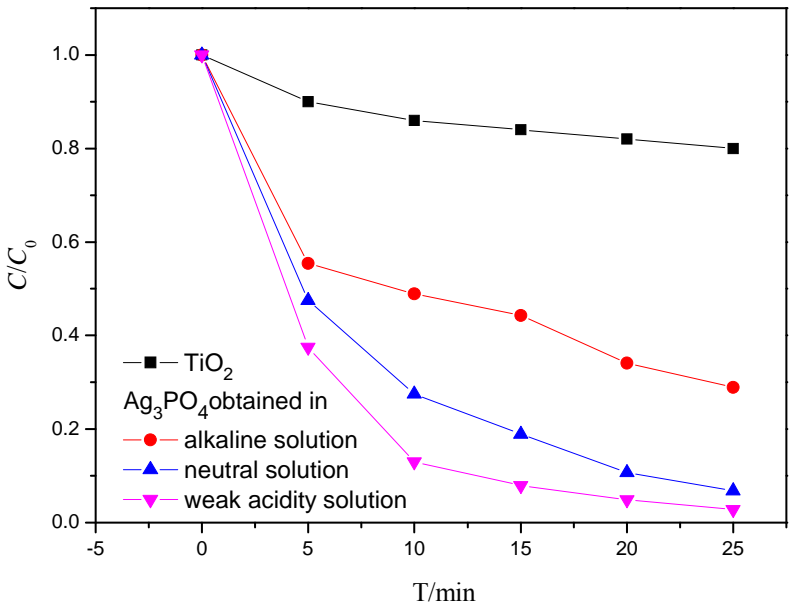

Fig.3. Degradation of MB over different photocatalysts

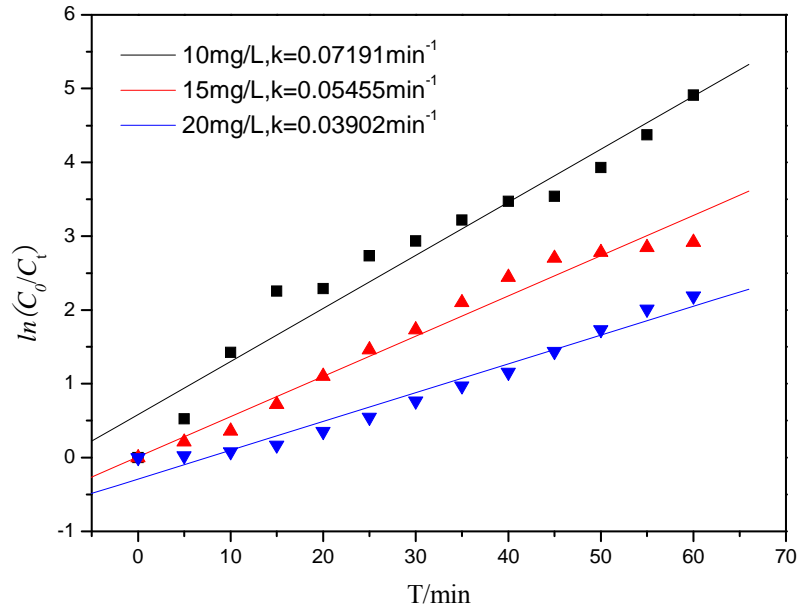

Fig.4. Pseudo-first-order kinetics of $\mathrm{MB}$ photodegradation process over $\mathrm{Ag}_{3} \mathrm{PO}_{4}$ samples.

The nature of dyes has significant influence on photocatalytic activities. As shown in Fig.5, it can be found that the photodegradation rate is much higher for the cationic dyes (MB) than that for the anionic dyes (MO) over $\mathrm{Ag}_{3} \mathrm{PO}_{4}$ product which maybe due to that $\mathrm{Ag}_{3} \mathrm{PO}_{4}$ product inherits negative surface charge and it makes for adsorbing the cationic dyes and promote the degradation reaction.

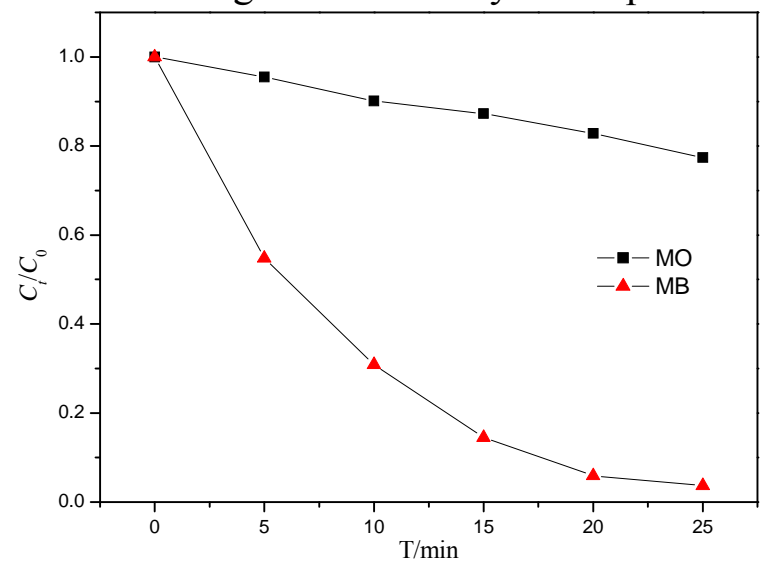

Fig.5. Photocatalytic degradation of different dyes in $\mathrm{Ag}_{3} \mathrm{PO}_{4}$ suspension
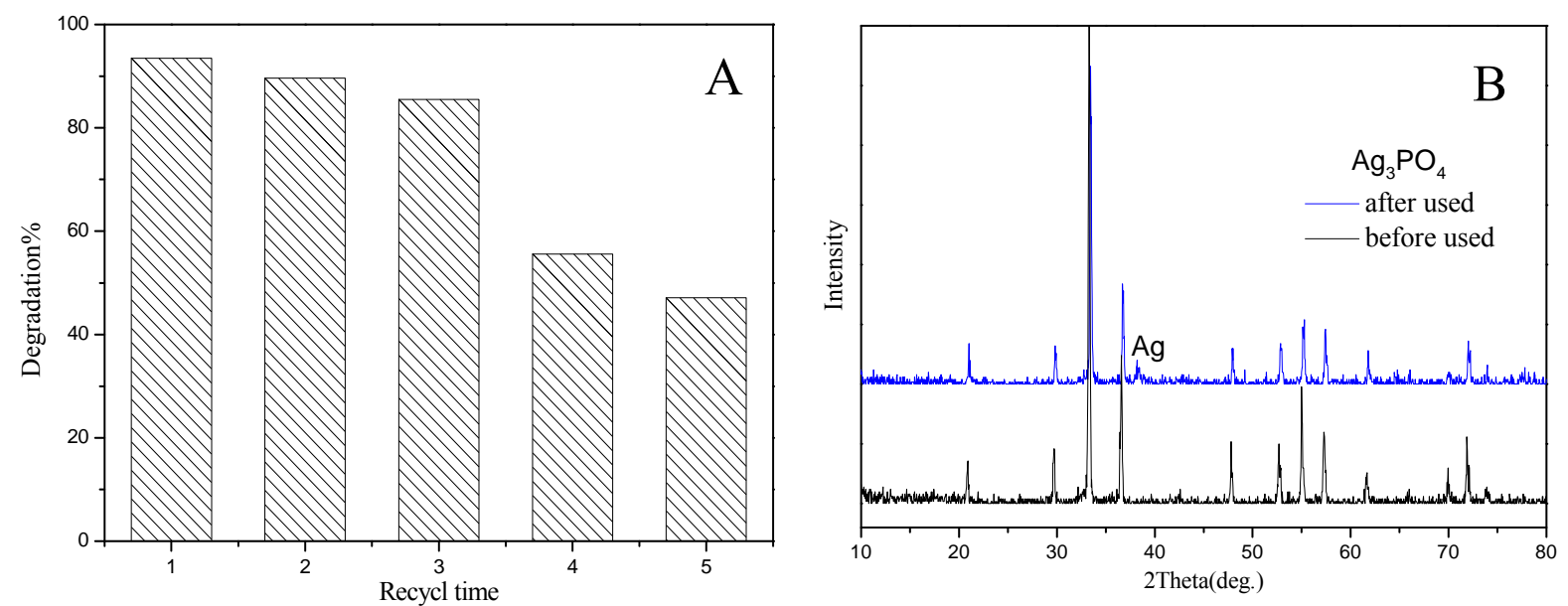

Fig.6. (A)The photodegradation rate of $\mathrm{MB}$ over $\mathrm{Ag}_{3} \mathrm{PO}_{4}$ in cycling runs;

(B)XRD patterns of $\mathrm{Ag}_{3} \mathrm{PO}_{4}$ photocatalyst before and after cycling runs. 
The recycle experiments were carried out to investigate the recyclability of as-synthesized $\mathrm{Ag}_{3} \mathrm{PO}_{4}$. The photodegradation rate of $\mathrm{MB}$ over $\mathrm{Ag}_{3} \mathrm{PO}_{4}$ on a number of cycling runs is shown in Fig.6. The degradation rate equation is as Eq. 2,

Degradation $\%=\left(C_{0}-C_{t}\right) / C_{0}$.

Fig.6(A) makes clear that the $\mathrm{Ag}_{3} \mathrm{PO}_{4}$ maintains high photocatalytic efficiency in the first three photocatalytic degradation process. After five cycles, the degradation efficiency change to half of the original. Fig.6(B) shows that the peak indexed to Ag can be found after the cycling runs, which indicates that part of the $\mathrm{Ag}_{3} \mathrm{PO}_{4}$ has decomposed to Ag. The Ag could cover the active sites on the surfaces of $\mathrm{Ag}_{3} \mathrm{PO}_{4}$ particles which results in the decrease of photocatalytic efficiency.

\section{Conclusions}

1. The silver phosphate photocatalyst was successfully synthesized by a simple ultrasonic-assisted ion-exchange method in weak acidity solution.

2. As-synthesized photocatalyst exhibited an excellent photocatalytic efficiency. Under visible light irradiation, nearly $100 \%$ of methylene blue $\left(10 \mathrm{mg} \cdot \mathrm{L}^{-1}, 50 \mathrm{~mL}\right)$ can be degraded by only $0.040 \mathrm{~g}$ catalyst in 25 minutes.

3. It was found out that the photodegradation rate of $\mathrm{MB}$ is higher when the initial concentration of dye is lower.

4. As-synthesized $\mathrm{Ag}_{3} \mathrm{PO}_{4}$ photocatalyst is more effective in photocatalytic degradation of the cationic dyes.

\section{Acknowledgements}

This work was financially supported by the Shaanxi Natural Science Foundation Research Project (2015JM2052).

\section{References}

[1] Ming Ge, Chinese Journal of Catalysis. Vol.35, 1410-1417. (2014)

[2] Xinguo Ma, Bin Lu, Di Li, Rui Shi, Chenshi Pan, and Yongfa Zhu, The Journal of Physical Chemistry C. Vol.115, 4680 - 4687. (2011)

[3] Yusuke Iida, Kazuhiko Akiyama, Journal of Alloys and Compounds. Vol.645, 1-6.(2015)

[4] Ming Ge, Mianmian Tan, Acta Physico-chemica Sinica. Vol.30, 2107-2112. (2014)

[5] Meirong Sui, Xiuquan Gu, Materials and Structures. 1671-4776. In Chinese (2013)

[6] Ming Ge, Chinese Journal of Catalysis. Vol.35, 1410-1417.(2014)

[7] A. Khana, M. Qamarb, M. Muneer, Chemical Physics Letters. Vol.519-520, 54-58.(2012)

[8] Lijun Huang: Synthesis and Photocatalytic Properties of silver Phosphate, Jilin University. In Chinese(2013)

[9] H.Williams, R. L. Withers, Nat. Mater. 9, 559-564.(2010)

[10] Yingpu Bi, Shuxin Ouyang, Naoto Umezawa, Junyu Cao, and Jinhua Ye, Journal of the American Chemical Society. Vol.133, 6490 - 6492.(2011)

[11] Ming Ge, Na Zhu, Yaping Zhao, Jing Li and Lu Liu, Industrial and Engineering Chemistry Research. Vol.51, 5167-5173.(2012) 